\title{
Molecular dynamics simulations of structural transformation of perfluorooctane sulfonate (PFOS) at water/rutile interfaces
}

\author{
Guangzhi He*, Meiyi Zhang, Qin Zhou, Gang Pan* \\ Department of Environmental Nano-materials, Research Center for Eco-Environmental Sciences, Chinese Academy of Sciences, Beijing 100085, China
}

\section{H I G H L I G H T S}

- Structures of PFOS assembled at the water-rutile interface were investigated.

- Concentration and salinity conditions significantly affect PFOS-solid interaction.

- $\mathrm{Ca}^{2+}$ enhanced the critical concentration for occurrence of multilayer adsorption.

- An atomic picture for the counterionbridging in PFOS adsorption was provided.

\section{A R T I C L E I N F O}

\section{Article history:}

Received 15 December 2014

Received in revised form 29 March 2015

Accepted 16 April 2015

Available online 15 May 2015

\section{Keywords:}

Perfluorinated compounds

Interfacial behavior

Assembling structure

Environmental factors

Concentration effect

Salinity effect
G R A P H I C A L A B S T R A C T

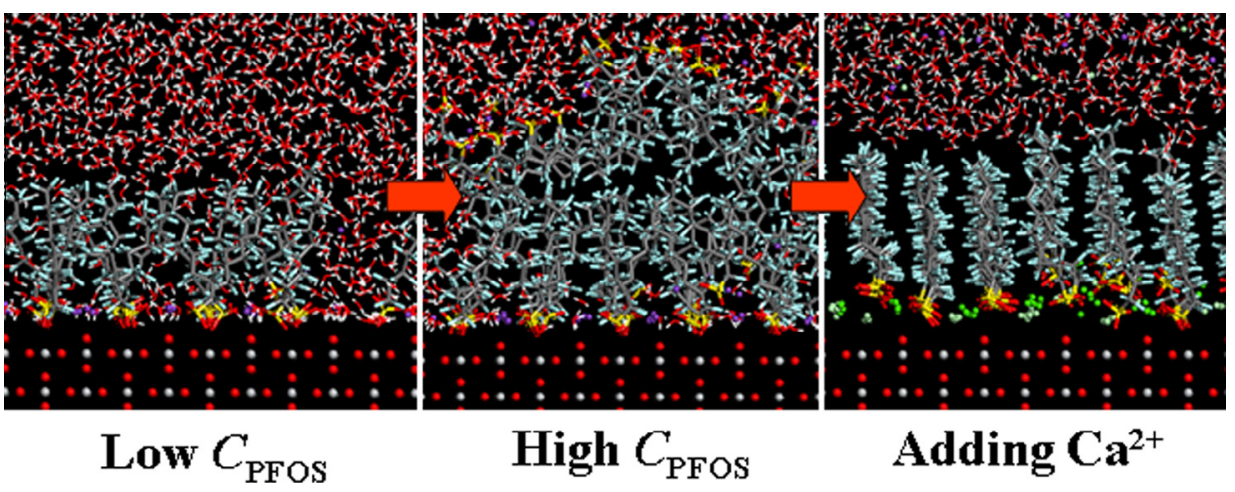

\begin{abstract}
A B S T R A C T
Concentration and salinity conditions are the dominant environmental factors affecting the behavior of perfluorinated compounds (PFCs) on the surfaces of a variety of solid matrices (suspended particles, sediments, and natural minerals). However, the mechanism has not yet been examined at molecular scales. Here, the structural transformation of perfluorooctane sulfonate (PFOS) at water/rutile interfaces induced by changes of the concentration level of PFOS and salt condition was investigated using molecular dynamics (MD) simulations. At low and intermediate concentrations all PFOS molecules directly interacted with the rutile (110) surface mainly by the sulfonate headgroups through electrostatic attraction, yielding a typical monolayer structure. As the concentration of PFOS increased, the molecules aggregated in a complex multi-layered structure, where an irregular assembling configuration was adsorbed on the monolayer structure by the van der Waals interactions between the perfluoroalkyl chains. When adding $\mathrm{CaCl}_{2}$ to the system, the multi-layered structure changed to a monolayer again, indicating that the addition of $\mathrm{CaCl}_{2}$ enhanced the critical concentration value to yield PFOS multilayer assemblies. The divalent $\mathrm{Ca}^{2+}$ substituted for monovalent $\mathrm{K}^{+}$as the bridging counterion in PFOS adsorption. MD simulation may trigger wide applications in study of perfluorinated compounds (PFCs) from atomic/molecular scale.
\end{abstract}

(C) 2015 Elsevier Ltd. All rights reserved.

\footnotetext{
* Corresponding authors.

E-mail addresses: gzhhe@rcees.ac.cn (G. He), myzhang@rcees.ac.cn (M. Zhang), qinzhou@rcees.ac.cn (Q. Zhou), gpan@rcees.ac.cn (G. Pan).
}

\section{Introduction}

Perfluorinated compounds (PFCs) occur globally in water and soil environments (Lau et al., 2007; Route et al., 2014). Perfluorooctane sulfonate (PFOS, $\mathrm{C}_{8} \mathrm{~F}_{17} \mathrm{SO}_{3}^{-}$) is the most commonly measured PFCs, and has been included in the list of Stockholm Convention on 
Persistent Organic Pollutants (POPs) in 2009 as a global contaminant (Wang et al., 2009). Different from other POPs, PFOS has high water solubility. The high persistence and long-range transport of PFOS in environments have prompted increasing concerns regarding its interfacial behavior that is regarded as central to the environmental fate of PFOS and the treatment of high-concentration wastewater (Pan and You, 2010; Xiao et al., 2011; Zhou et al., 2013).

So far, although PFOS has been investigated extensively, little is known about its interfacial microstructure and the interaction that hold PFOS to the surface because it is inaccessible from the existing experimental measurement (Du et al., 2014). However, the microscopic information is essential to enhance our understanding of the environmental fate of PFOS and to develop high performance adsorbents, because the change of assembling structure and interaction may produce a significant impact on its reversibility, lability, and transport in the environment and the efficiency of water treatment. Although the critical micelle concentration (CMC) of PFOS is $4573 \mathrm{mg} \mathrm{L}^{-1}$, it is possible to form hemi-micelles on adsorbent surfaces when its concentration reaches the value of 0.01-0.001 of the CMC (Johnson et al., 2007) (a concentration level of PFOS in experiments and water treatment systems). Therefore, increase of PFOS concentrations promotes the nucleation of hemi-micelles and micelles (Yu et al., 2009; Wang and Shih, 2011), which may result in a substantial increase of PFOS at the interface, and a significant change in the macroscopic sorption properties (e.g., the occurrence of multilayer adsorption (Zhao et al., 2011)). Different types of hemi-micelles and multi-layered structures have been proposed based on macroscopic experiments (Chen et al., 2011; Zhang et al., 2011b; Du et al., 2014). However, these structures need to be identified at molecular scales.

PFOS is an anionic surfactant, and hence the ionic strength and cation type in solutions are critical in the adsorption of PFOS on solid surfaces (Jeon et al., 2010; You et al., 2010; Wang and Shih, 2011). Different types of cations may yield very different effects on both interaction strength and structure. However, most sorption experiments provide only macroscopic information (e.g., sorption capacity and sorption kinetics) about the interfacial behavior of PFOS, but little insight into the interaction and microstructure. In fact, changes in the sorption isotherms driven by concentration and salinity conditions depend on the interactions between hydrophobic tails, repulsions between headgroups and interactions between surfactant molecules with the solid surface (Yu et al., 2009; Wang and Shih, 2011). These mechanisms are required to be verified by using atomic-molecular level technologies.

Molecular dynamics (MD) simulation, which solves the movement of atoms and molecules, provides detailed insights pertaining to structure, energetics, and dynamics of complex multiphase systems. MD simulation has been successfully used to investigate the interfacial behavior of alkyl surfactants, such as sodium dodecyl sulfate (SDS) and n-alkyl polyethylene oxide (Srinivas et al.,

Table 1

Partial atomic charges used to model the assembly of PFOS at the water-rutile interface.

\begin{tabular}{lc}
\hline Atom & Charges/e \\
\hline $\mathrm{S}$ & 1.474 \\
$\mathrm{O}\left(\right.$ in $\left.\mathrm{SO}_{3}\right)$ & -0.706 \\
$\mathrm{C}\left(\right.$ in $\left.\mathrm{S}_{-} \mathrm{CF}_{2}\right)$ & 0.166 \\
$\mathrm{C}\left(\right.$ in $\left.\mathrm{CF}_{2}-\mathrm{CF}_{2}\right)$ & 0.520 \\
$\mathrm{C}\left(\right.$ in $\left.\mathrm{CF}_{3}\right)$ & 0.780 \\
$\mathrm{~F}$ & -0.260 \\
$\mathrm{~K}$ & 1.000 \\
$\mathrm{Ti}$ & 1.600 \\
$\mathrm{O}\left(\right.$ in $\left.\mathrm{TiO}_{2}\right)$ & -0.800 \\
$\mathrm{O}\left(\right.$ in $\left.\mathrm{H}_{2} \mathrm{O}\right)$ & -0.820 \\
$\mathrm{H}$ & 0.410 \\
\hline
\end{tabular}

Table 2

Initial structure of PFOS molecule*.

\begin{tabular}{llll}
\hline Distance & Value $(\AA)$ & Angle & Value $\left(^{\circ}\right)$ \\
\hline S-O & 1.48 & S-C-C & 116 \\
S-C & 1.91 & C-C-C & 114 \\
C-C & 1.57 & & \\
C-F & 1.36 & & \\
\hline
\end{tabular}

The structural parameters are from the DFT-calculated data in the literature (Torres et al., 2009).

2006; Tummala and Striolo, 2008; Dominguez, 2011; Lin et al., 2011; Nunez-Rojas and Dominguez, 2011). Compared with alkyl surfactants, the perfluoroalkyl surfactant may present different assembling features due to the super hydrophobicity and counterion-bridging effects. To our knowledge, how the environmental factors such as concentration and salinity affect the structure and behavior of perfluorinated compounds (PFCs) at solid-water interfaces has not yet been investigated at molecular scales.

$\mathrm{TiO}_{2}$ is one of the most common and technologically important metal oxides for modern science and technology, and shows a relatively high affinity to PFOS (Yuan et al., 2001). Rutile $\mathrm{TiO}_{2}$ was chosen as the model oxide surface in this MD study. This study is expected to provide an effective way to investigate the microstructures and interaction properties of PFOS at water-metal oxide (e.g., $\mathrm{Fe}-, \mathrm{Al}-, \mathrm{Mn}-$, and Ti-oxides) interfaces, and may trigger wide applications of MD simulation in describing the structure and environmental behavior of PFCs as MD technique is improving.

Here, the structural transformation and interaction properties of PFOS assembled at the water-rutile interface as a function of concentration and salinity conditions were studied using molecular dynamics (MD) simulation. The surface aggregation was characterized in terms of structure, shape, and associated mode. Effects of salinity on the surface assembling properties of PFOS were investigated by adding $\mathrm{CaCl}_{2}$ to the simulated system. The conformational feature of PFOS was described using the atomic density profile and angle distribution analysis. The role of counterions $\left(\mathrm{K}^{+}, \mathrm{Ca}^{2+}\right)$ in the formation of surface aggregates was characterized with the radial distribution functions between atoms.

\section{Methods}

All simulations were carried out using the COMPASS (Condensed-phase Optimized Molecular Potentials for Atomistic Simulation Studies) force field as implemented in the Discover module in Materials Studio package (Accelrys Software Inc.). The functional form of COMPASS force field is given as follows (Sun, 1998; Zhang et al., 2011a):

$$
\begin{aligned}
E_{p o t}= & \sum_{b}\left[K_{2}\left(b-b_{0}\right)^{2}+K_{3}\left(b-b_{0}\right)^{3}+K_{4}\left(b-b_{0}\right)^{2}\right] \\
& +\sum_{\theta}\left[H_{2}\left(\theta-\theta_{0}\right)^{2}+H_{3}\left(\theta-\theta_{0}\right)^{3}+H_{4}\left(\theta-\theta_{0}\right)^{2}\right] \\
& +\sum_{\phi}\left[V_{1}\left(1-\cos \left(\phi-\phi_{1}^{0}\right)\right)+V_{2}\left(1-\cos \left(2 \phi-\phi_{2}^{0}\right)\right)\right. \\
& \left.+V_{3}\left(1-\cos \left(3 \phi-\phi_{3}^{0}\right)\right)\right] \\
& +\sum_{\chi} K_{\chi}\left(\chi-\chi_{0}\right)^{2} \\
& +\sum_{b} \sum_{b^{\prime}} F_{b b^{\prime}}\left(b-b_{0}\right)\left(b^{\prime}-b_{0}^{\prime}\right) \\
& +\sum_{\theta} \sum_{\theta^{\prime}} F_{\theta \theta^{\prime}}\left(\theta-\theta_{0}\right)\left(\theta^{\prime}-\theta_{0}^{\prime}\right)
\end{aligned}
$$






a

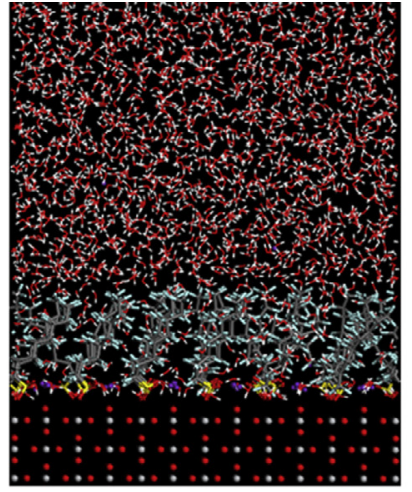

b

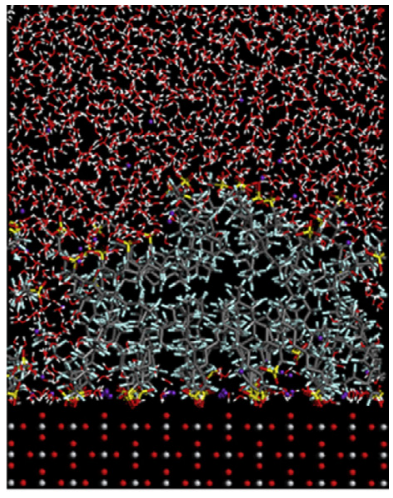

C

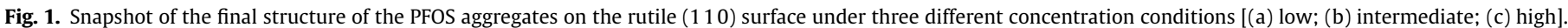

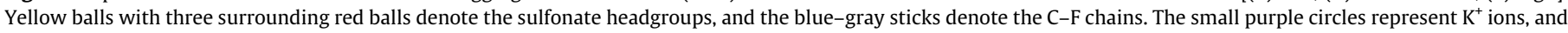

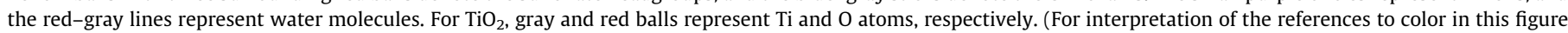
legend, the reader is referred to the web version of this article.)

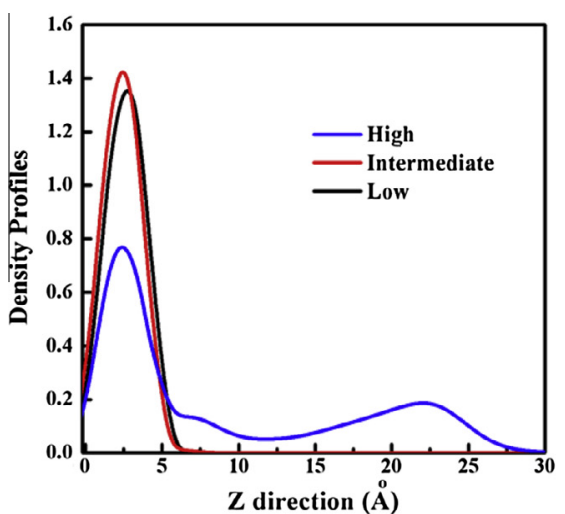

a

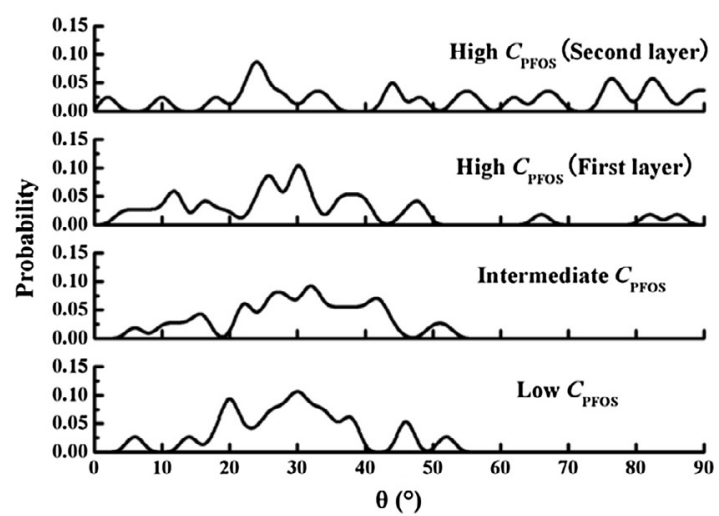

b

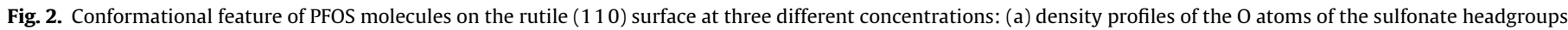
along the $Z$ direction; and (b) angle distribution of PFOS with respect to the surface normal.

$$
\begin{aligned}
& +\sum_{b} \sum_{\theta} F_{b \theta}\left(b-b_{0}\right)\left(\theta-\theta_{0}\right) \\
& +\sum_{b} \sum_{\phi}\left(b-b_{0}\right)\left(V_{1} \cos \phi+V_{2} \cos 2 \phi+V_{3} \cos 3 \phi\right) \\
& +\sum_{b^{\prime}} \sum_{\phi}\left(b^{\prime}-b_{0}^{\prime}\right)\left(V_{1} \cos \phi+V_{2} \cos 2 \phi+V_{3} \cos 3 \phi\right) \\
& +\sum_{\theta} \sum_{\phi}\left(\theta-\theta_{0}\right)\left(V_{1} \cos \phi+V_{2} \cos 2 \phi+V_{3} \cos 3 \phi\right) \\
& +\sum_{\phi} \sum_{\theta} \sum_{\theta^{\prime}} K_{\phi \theta \theta^{\prime}} \cos \phi\left(\theta-\theta_{0}\right)\left(\theta^{\prime}-\theta_{0}^{\prime}\right) \\
& +\sum_{i>j} \frac{q_{i} q_{j}}{\varepsilon r_{i j}} \\
& +\sum_{i>j}\left[\frac{A_{i j}}{r_{i j}^{9}}-\frac{B_{i j}}{r_{i j}^{6}}\right]
\end{aligned}
$$

The total potential energy $\left(E_{\text {pot }}\right)$ contains the bond stretching (a), angle bending (b), torsion (c), out of plane coordinate (d), cross-coupling (e)-(k), Coulombic interaction (l), and van der Waals interaction (m) terms. $K, H, F$, and $V$ are force-field parameters. The $b, \theta, \varphi$, and $\chi$ represent bond length, bending angle, torsion angle, and out-of-plane angle, respectively. The parameters $b_{0}, \theta_{0}, \varphi_{0}$, and $\chi_{0}$ are the ideal values at zero energy. COMPASS has proven to be suitable for simulating titanium oxides (Kornherr et al., 2004, 2006; Zhang et al., 2011a), surfactants (Ryjkina et al., 2002), and perfluorinated compounds (Prathab et al., 2006; Li et al., 2013). The agreement between our calculated PFOS structure and the DFT-calculated results (see Supplementary Data) indicated that the COMPASS force field is reliable for the description of PFOS. Partial atomic charges used in COMPASS are presented in Table 1.

The (110) crystal plane was used as the surface because it is the predominantly exposed plane of natural rutile (60\%) (Perron et al., 2007). The dimensions of the solid model were $4.1 \times 3.9 \times 1.3 \mathrm{~nm}^{3}$, which contained $766 \mathrm{TiO}_{2}$ molecules. The initial configuration of the interfacial system was prepared from a monolayer of PFOS molecules (a sulfonate headgroup attached to eight perfluorinated carbon atoms with a length of $\sim 1.0 \mathrm{~nm}$ ) with the sulfonate headgroups pointed to the surface ( $\sim 8 \AA$ above the substrate). The initial structure of PFOS is shown in Table $2 . \mathrm{K}^{+}$ions were added close to the headgroups to neutralize the charge of anionic PFOS (i.e., the potassium salt of PFOS). To reach a water density of $\sim 1.0 \mathrm{~g} / \mathrm{cc}$ within the solution layer of $4 \times 4 \times 4 \mathrm{~nm}^{3}, 2000$ water molecules were used to simulate the solvent environment. The water was simulated using the simple point charge (SPC) model (Berendsen et al., 1987), which worked reliably with the COMPASS force field. 


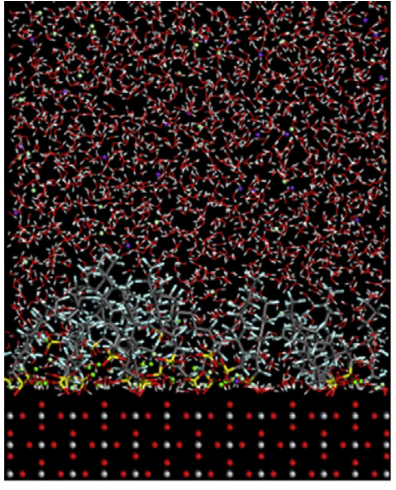

a

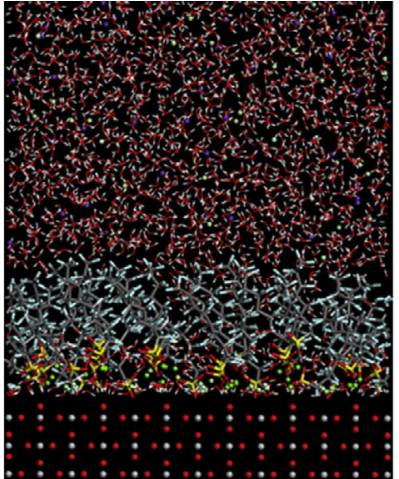

b

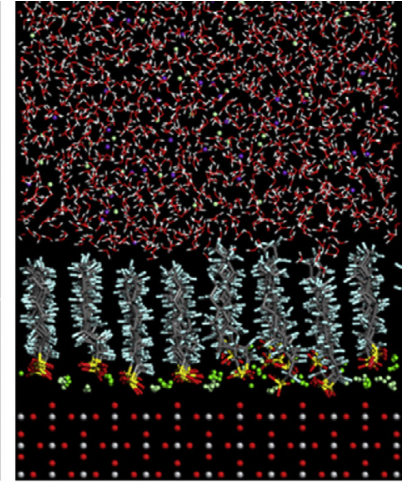

c

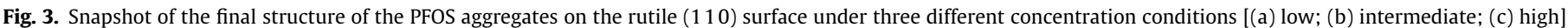

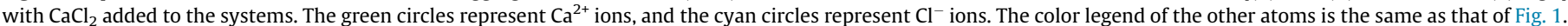
(For interpretation of the references to color in this figure legend, the reader is referred to the web version of this article.)

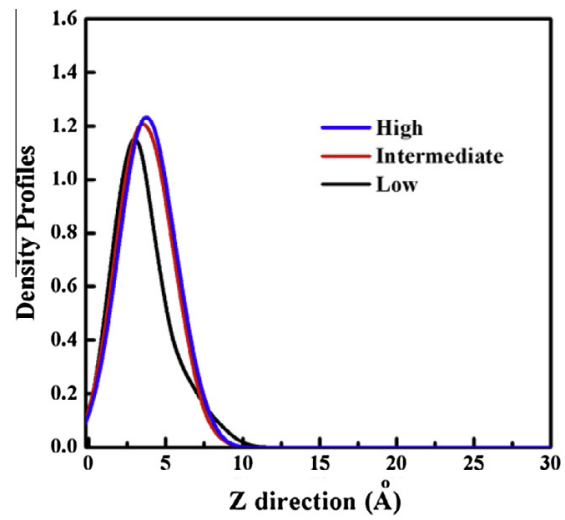

a

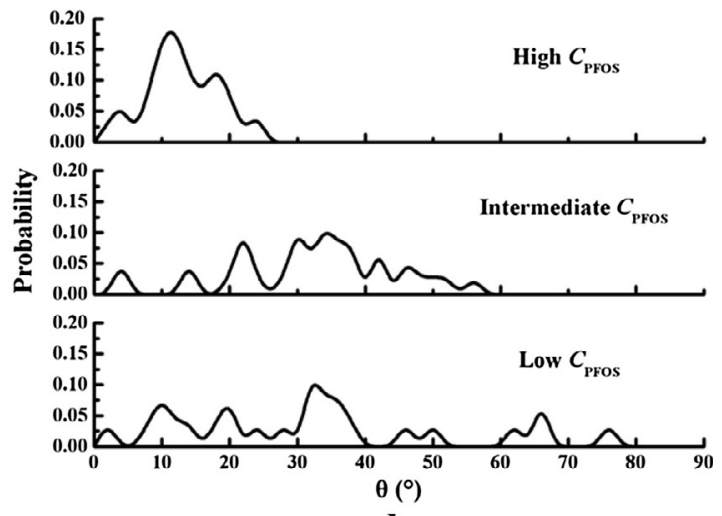

b

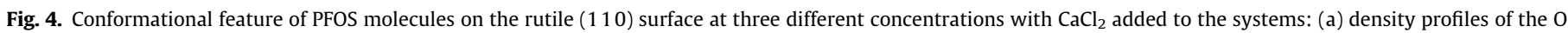
atoms of the sulfonate headgroups along the $Z$ direction; and (b) angle distribution of PFOS with respect to the surface normal.

The atoms in rutile were constrained to their equilibrium bulk positions, while the structures of PFOS and water molecules were optimized during the simulations ( $\mathrm{Li}$ and Choi, 2007; Prathab et al., 2007; Nunez-Rojas and Dominguez, 2011). Periodic boundary conditions were imposed, and these systems were separated from their periodic images by a vacuum gap of $6 \mathrm{~nm}$ to eliminate spurious interactions between the periodic replicas in the $Z$ direction (Dominguez, 2009; Monti et al., 2012). The resulted final box was approximately $4 \times 4 \times 12 \mathrm{~nm}^{3}$.

The simulation was performed under three different PFOS concentrations ( 25,36 , and 64 PFOS molecules) to investigate how the concentration levels affected the interfacial assembling behavior of PFOS. In order to explore the salinity effects, a certain number of $\mathrm{Ca}^{2+}$ and $\mathrm{Cl}^{-}$were added to the systems to achieve a ratio of PFOS to $\mathrm{CaCl}_{2}$ being $1: 1$. The systems were first energetically minimized for 5000 steps using the smart minimizer method, which switches from steepest-descent to conjugated gradient method as the energy derivatives decrease to accelerate the computation. After the initialization, all the MD simulations were conducted in the canonical ensemble (NVT) at a constant temperature of $298 \mathrm{~K}$ maintained using Andersen thermostat (Andersen, 1980). The equations of motion were integrated with the velocity Verlet algorithm with a time step of 1 fs (Verlet, 1967). The electrostatic interactions were treated using the Ewald method (Plimpton, 1995), and the Van der Waals interactions were handled with atom-based summation method using a cutoff distance of $9.5 \AA$ A with long-range corrections added. The simulations were confirmed to have reached equilibrium within 15 ns by monitoring the structure and shape of PFOS aggregates as a function of time. Therefore, all the systems were finally equilibrated for 18 ns.

The selection of parameters and models was justified by performing test calculations (see Supplementary Data). Increasing the orbital cutoff from 9.5 to $12.0 \AA$ had no obvious effect on the interfacial structure and properties of PFOS. Similarly, no obvious change was found in the equilibrium structure when we started the simulation with a different initial configuration (i.e., the PFOS molecules parallel to the surface), indicating that the MD results do not depend on the initial conditions. These tests verified that the present computational settings and models were reliable for describing the structure and properties of PFOS at water-rutile interface.

\section{Results and discussions}

\subsection{Concentration effects}

The equilibrium structures of PFOS assembled at the water-rutile interface under three different (low, intermediate, and high) concentration conditions are present in Fig. 1. A structural transformation driven by an increment of PFOS concentration was clearly identified. At the low and intermediate concentrations, all PFOS molecules directly interacted with the rutile (110) surface mainly by the sulfonate headgroups through electrostatic attraction, and 


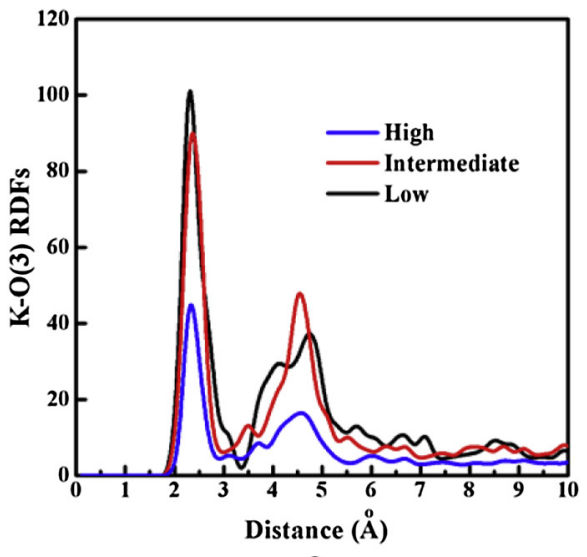

a

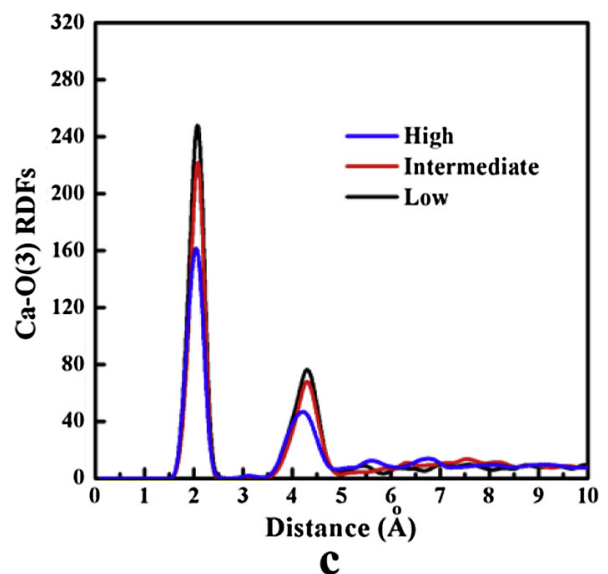

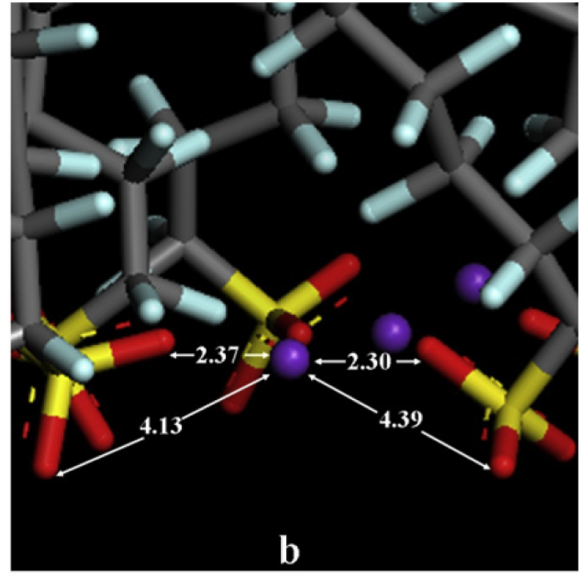

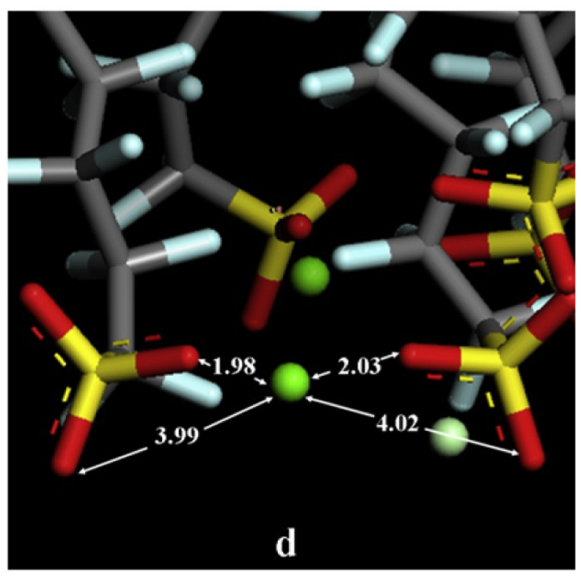

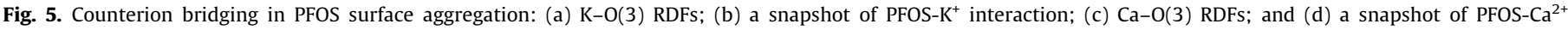

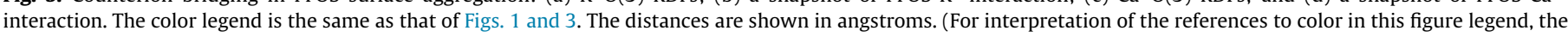
reader is referred to the web version of this article.)

well arrayed in a monolayer (Fig. 1a and b). It was observed that the sulfonate headgroups close to the solid surfaces were linked together by the $\mathrm{K}^{+}$ions, leaving the perfluoroalkyl tails away from the surface. As the concentration increased, PFOS molecules arrayed in a different pattern, where a number of PFOS molecules were adsorbed on the monolayer structure by the van der Waals interactions between the perfluoroalkyl chains, forming a multilayer aggregate (Fig. 1c).

The PFOS-surface interaction was depicted from the density profiles of the $\mathrm{O}$ atoms of the sulfonate headgroups in the $Z$-direction (i.e. normal to the solid surfaces, Fig. 2a). The orientation of PFOS molecules at the water-rutile interface was determined by the angle $(\theta)$ between the $\mathrm{C} 1-\mathrm{C} 8$ vector ( $\mathrm{C} 1$ is the $\mathrm{C}$ atom attached to the sulfonate headgroup, and $\mathrm{C} 8$ is the $\mathrm{C}$ atom at the end of the perfluoroalkyl chain) and the surface normal (Fig. 2b). The density profiles showed that the sulfonate headgroups interacted with the rutile surfaces mainly at the distance of $2.0-3.5 \AA$ (see the main peaks in Fig. 2a). At low and intermediate concentrations, more than $85 \%$ of PFOS molecules were located on the surface with the angle $\theta$ less than $45^{\circ}$ (Fig. 2b), indicating that most of the PFOS preferred to align perpendicular to the substrate. This conformation is favorable for the electrostatic interaction between the sulfonate headgroups and the solid surface. At high concentration the second adsorbed layer was observed, corresponded to the peaks of the sulfonate headgroups at 17.5-25.0 ̊ in Fig. 2a. In the second layer, the PFOS molecules were inclined to the surface with an angle range from $0^{\circ}$ to $90^{\circ}$ (Fig. 2b) and hence formed an irregular assembling configuration (Fig. 1c).

\subsection{Salinity effects}

When $\mathrm{Ca}^{2+}$ ions were added to the system, PFOS displayed a different assembling behavior in terms of the morphology and layer formation (Figs. 3 and 4a). The angles $\theta$ became smaller as the PFOS concentration increased (Fig. 4b), indicating that the PFOS monolayer underwent an ordering transformation. When $\mathrm{Ca}^{2+}$ was present, PFOS molecules were inclined to the surface with an angle range from $0^{\circ}$ to $80^{\circ}$ and formed a relatively irregular monolayer structure at the low concentration, whereas arrayed in a well-ordered monolayer pattern with the perfluoroalkyl chains nearly perpendicular to the substrate (all the angles $\theta$ less than $30^{\circ}$ ) under the high concentration condition. At high PFOS concentration, the multi-layered structure (Fig. 1c) changed to a monolayer again when adding $\mathrm{CaCl}_{2}$ (Fig. 3c), indicating that the addition of $\mathrm{CaCl}_{2}$ enhanced the critical concentration value for the occurrence of PFOS multilayer adsorption.

\subsection{Counterion-bridging effects}

To identify the role of the counterions $\left(\mathrm{K}^{+}\right.$and $\left.\mathrm{Ca}^{2+}\right)$ in the interfacial processes of PFOS, the $\mathrm{K}-\mathrm{O}(3)$ and $\mathrm{Ca}-\mathrm{O}(3)[\mathrm{O}(3)$, the $\mathrm{O}$ atoms on the $-\mathrm{SO}_{3}$ headgroups] radial distribution functions (RDFs) were analyzed (Fig. 5a and c). An enlarged snapshot of PFOS interacted with $\mathrm{K}^{+}$and $\mathrm{Ca}^{2+}$ are present in Fig. 5b and $\mathrm{d}$, which provided an atomic picture for counterion-bridging mechanism in PFOS adsorption. As shown in Fig. 5b, the $\mathrm{K}^{+}$associated with the sulfonate headgroups of PFOS molecules at the surfaces, 
reducing the lateral repulsive force between the anionic sulfonate groups and instead inducing an effective attraction. When $\mathrm{CaCl}_{2}$ was added to the system, the divalent $\mathrm{Ca}^{2+}$ substituted for monovalent $\mathrm{K}^{+}$as the bridging counterion (Fig. 5d). The RDFs were dominated by two peaks at $\sim 2.3$ and $\sim 4.3 \AA$ for PFOS- $\mathrm{K}^{+}$interaction while at $\sim 2.0$ and $\sim 4.1 \AA$ for $\mathrm{PFOS}_{-} \mathrm{Ca}^{2+}$ interaction (Fig. $5 \mathrm{a}$ and $\mathrm{c}$ ), which corresponded to the distances between the $\mathrm{K}^{+} / \mathrm{Ca}^{2+}$ and the neighbor and non-neighbor $\mathrm{O}$ atoms of two adjacent sulfonate groups (Fig. $5 \mathrm{~b}$ and d). These results of RDFs indicated that the salinity conditions imposed a difference towards the surface aggregation of PFOS, and the highly charged $\mathrm{Ca}^{2+}$ led to stronger interaction with the sulfonate groups.

\section{Conclusions}

The molecular dynamics (MD) results indicated that concentration and salinity conditions significantly affected the assembling behavior of PFOS at the solid-water interface. At low and intermediate concentrations, PFOS molecules directly interacted with the rutile surface mainly by the sulfonate headgroups through electrostatic attraction, and aggregated in a regular monolayer structure. As the concentration increased, PFOS molecules arrayed in a different pattern, where an irregular assembling configuration was adsorbed on the monolayer structure by the van der Waals interactions between the perfluoroalkyl chains, forming a multilayer aggregate. When adding $\mathrm{CaCl}_{2}$ to the system, the multi-layered structure changed to a monolayer again, indicating that the addition of $\mathrm{CaCl}_{2}$ enhanced the critical concentration value for the occurrence of PFOS multilayer adsorption. An atomic picture for the counterion-bridging in PFOS adsorption was provided, where the highly charged $\mathrm{Ca}^{2+}$ substituted for $\mathrm{K}^{+}$as the bridging ion to link the sulfonate groups and thus caused the occurrence of surface aggregation. MD simulation provides new perspective for the sorptive characteristics of PFOS, and may trigger wide applications in study of perfluorinated compounds (PFCs) from atomic/molecular scale.

\section{Acknowledgments}

The study was supported by NNSF of China (21207151, 21377003, and 41103076). We thank the Supercomputing Center of the Chinese Academy of Sciences for providing access to the Materials Studio software.

\section{Appendix A. Supplementary material}

The validation of computational settings and models. Supplementary data associated with this article can be found, in the online version, at http://dx.doi.org/10.1016/j.chemosphere. 2015.04.056.

\section{References}

Andersen, H.C., 1980. Molecular dynamics simulations at constant pressure and/or temperature. J. Chem. Phys. 72, 2384-2393.

Berendsen, H.J.C., Grigera, J.R., Straatsma, T.P., 1987. The missing term in effective pair potentials. J. Phys. Chem. 91, 6269-6271.

Chen, X., Xia, X., Wang, X., Qiao, J., Chen, H., 2011. A comparative study on sorption of perfluorooctane sulfonate (PFOS) by chars, ash and carbon nanotubes. Chemosphere 83, 1313-1319.

Dominguez, H., 2009. Structure of the sodium dodecyl sulfate surfactant on a solid surface in different $\mathrm{NaCl}$ solutions. Langmuir 25, 9006-9011.

Dominguez, H., 2011. Structural transition of the sodium dodecyl sulfate (SDS) surfactant induced by changes in surfactant concentrations. J. Phys. Chem. B $115,12422-12428$

Du, Z., Deng, S., Bei, Y., Huang, Q., Wang, B., Huang, J., Yu, G., 2014. Adsorption behavior and mechanism of perfluorinated compounds on various adsorbents-a review. J. Hazard. Mater. 274, 443-454.
Jeon, J., Kannan, K., Lim, H.K., Moon, H.B., Ra, J.S., Kim, S.D., 2010. Bioaccumulation of perfluorochemicals in Pacific oyster under different salinity gradients. Environ. Sci. Technol. 44, 2695-2701.

Johnson, R.L., Anschutz, A.J., Smolen, J.M., Simcik, M.F., Penn, R.L., 2007. The adsorption of perfluorooctane sulfonate onto sand, clay, and iron oxide surfaces. J. Chem. Eng. Data 52, 1165-1170.

Kornherr, A., Tortschanoff, A., Portuondo-Campa, E., van Mourik, F., Chergui, M., Zifferer, G., 2006. Modelling of aqueous solvation of eosin Y at the rutile $\mathrm{TiO}_{2}(110) /$ water interface. Chem. Phys. Lett. 430, 375-379.

Kornherr, A., Vogtenhuber, D., Ruckenbauer, M., Podloucky, R., Zifferer, G., 2004. Multilayer adsorption of water at a rutile $\mathrm{TiO}_{2}(110)$ surface: towards a realistic modeling by molecular dynamics. J. Chem. Phys. 121, 3722-3726.

Lau, C., Anitole, K., Hodes, C., Lai, D., Pfahles-Hutchens, A., Seed, J., 2007. Perfluoroalkyl acids: a review of monitoring and toxicological findings. Toxicol. Sci. 99, 366-394.

Li, C., Choi, P., 2007. Molecular dynamics study of the adsorption behavior of normal alkanes on a relaxed alpha- $\mathrm{Al}_{2} \mathrm{O}_{3}(0001)$ surface. J. Phys. Chem. C 111, $1747-$ 1753.

Li, Y., Niu, J., Shen, Z., Feng, C., 2013. Size effect of single-walled carbon nanotube on adsorption of perfluorooctanesulfonate. Chemosphere 91, 784-790.

Lin, S., Shih, C.-J., Strano, M.S., Blankschtein, D., 2011. Molecular insights into the surface morphology, layering structure, and aggregation kinetics of surfactantstabilized graphene dispersions. J. Am. Chem. Soc. 133, 12810-12823.

Monti, S., van Duin, A.C.T., Kim, S.-Y., Barone, V., 2012. Exploration of the conformational and reactive dynamics of glycine and diglycine on $\mathrm{TiO}_{2}$ : computational investigations in the gas phase and in solution. J. Phys. Chem. C $116,5141-5150$.

Nunez-Rojas, E., Dominguez, H., 2011. Computational studies on the behavior of sodium dodecyl sulfate (SDS) at $\mathrm{TiO}_{2}$ (rutile)/water interfaces. J. Colloid Interface Sci. 364, 417-427.

Pan, G., You, C., 2010. Sediment-water distribution of perfluorooctane sulfonate (PFOS) in Yangtze River estuary. Environ. Pollut. 158, 1363-1367.

Perron, H., Domain, C., Roques, J., Drot, R., Simoni, E., Catalette, H., 2007. Optimisation of accurate rutile $\mathrm{TiO}_{2}$ (110), (100), (101) and (001) surface models from periodic DFT calculations. Theor. Chem. Acc. 117, 565-574.

Plimpton, S., 1995. Fast parallel algorithms for short-range molecular dynamics. J. Comput. Phys. 117, 1-19.

Prathab, B., Aminabhavi, T.M., Parthasarathi, R., Manikandan, P., Subramanian, V., 2006. Molecular modeling and atomistic simulation strategies to determine surface properties of perfluorinated homopolymers and their random copolymers. Polymer 47, 6914-6924.

Prathab, B., Subramanian, V., Aminabhavi, T.M., 2007. Molecular dynamics simulations to investigate polymer-polymer and polymer-metal oxide interactions. Polymer 48, 409-416.

Route, W.T., Key, R.L., Russell, R.E., Lindstrom, A.B., Strynar, M.J., 2014. Spatial and temporal patterns in concentrations of perfluorinated compounds in bald eagle nestlings in the Upper Midwestern United States. Environ. Sci. Technol. 48, 6653-6660.

Ryjkina, E., Kuhn, H., Rehage, H., Muller, F., Peggau, J., 2002. Molecular dynamic computer simulations of phase behavior of non-ionic surfactants. Angew. Chem. Int. Ed. 41, 983-986.

Srinivas, G., Nielsen, S.O., Moore, P.B., Klein, M.L., 2006. Molecular dynamics simulations of surfactant self-organization at a solid-liquid interface. J. Am. Chem. Soc. $128,848-853$.

Sun, H., 1998. COMPASS: an ab initio force-field optimized for condensed-phase applications - overview with details on alkane and benzene compounds. J. Phys. Chem. B 102, 7338-7364.

Torres, F.J., Ochoa-Herrera, V., Blowers, P., Sierra-Alvarez, R., 2009. Ab initio study of the structural, electronic, and thermodynamic properties of linear perfluorooctane sulfonate (PFOS) and its branched isomers. Chemosphere 76, $1143-1149$.

Tummala, N.R., Striolo, A., 2008. Role of counterion condensation in the selfassembly of SDS surfactants at the water-graphite interface. J. Phys. Chem. B $112,1987-2000$

Verlet, L., 1967. Computer experiments on classical fluids. 1. Thermodynamical properties of Lennard-Jones molecules. Phys. Rev. 159, 98-103.

Wang, F., Shih, K., 2011. Adsorption of perfluorooctanesulfonate (PFOS) and perfluorooctanoate (PFOA) on alumina: influence of solution $\mathrm{pH}$ and cations. Water Res. 45, 2925-2930.

Wang, T., Wang, Y., Liao, C., Cai, Y., Jiang, G., 2009. Perspectives on the inclusion of perfluorooctane sulfonate into the Stockholm Convention on persistent organic pollutants. Environ. Sci. Technol. 43, 5171-5175.

Xiao, F., Zhang, X., Penn, L., Gulliver, J.S., Simcik, M.F., 2011. Effects of monovalent cations on the competitive adsorption of perfluoroalkyl acids by kaolinite: experimental studies and modeling. Environ. Sci. Technol. 45, 10028-10035.

You, C., Jia, C., Pan, G., 2010. Effect of salinity and sediment characteristics on the sorption and desorption of perfluorooctane sulfonate at sediment-water interface. Environ. Pollut. 158, 1343-1347.

Yu, Q., Zhang, R., Deng, S., Huang, J., Yu, G., 2009. Sorption of perfluorooctane sulfonate and perfluorooctanoate on activated carbons and resin: kinetic and isotherm study. Water Res. 43, 1150-1158.

Yuan, Q.Z., Ravikrishna, R., Valsaraj, K.T., 2001. Reusable adsorbents for dilute solution separation. 5. Photodegradation of organic compounds on surfactantmodified titania. Sep. Purif. Technol. 24, 309-318.

Zhang, H., Lu, X., Leng, Y., Watari, F., Weng, J., Feng, B., Qu, S., 2011a. Effects of aqueous environment and surface defects on Arg-Gly-Asp peptide adsorption 
on titanium oxide surfaces investigated by molecular dynamics simulation. J. Biomed. Mater. Res. A 96A, 466-476.

Zhang, Q., Deng, S., Yu, G., Huang, J., 2011b. Removal of perfluorooctane sulfonate from aqueous solution by crosslinked chitosan beads: sorption kinetics and uptake mechanism. Bioresour. Technol. 102, 2265-2271.
Zhao, D., Cheng, J., Vecitis, C.D., Hoffmann, M.R., 2011. Sorption of perfluorochemicals to granular activated carbon in the presence of ultrasound. J. Phys. Chem. A 115, 2250-2257.

Zhou, Q., Pan, G., Zhang, J., 2013. Effective sorption of perfluorooctane sulfonate (PFOS) on hexadecyltrimethylammonium bromide immobilized mesoporous $\mathrm{SiO}_{2}$ hollow sphere. Chemosphere 90, 2461-2466. 